\title{
ANÁLISE EXPERIMENTAL E DE CFD DO ESCOAMENTO DE DIFERENTES MATERIAIS EM TAMBOR ROTATÓRIO COM SUSPENSORES
}

\author{
F.P. de LIMA ${ }^{1}$, L.F.G.de ÁVILA ${ }^{1}$, S.M. NASCIMENTO ${ }^{1}$, M.A.S. BARROZO ${ }^{1}$ e C.R. \\ DUARTE ${ }^{1}$ \\ ${ }^{1}$ Universidade Federal de Uberlândia, Faculdade de Engenharia Química \\ E-mail para contato: suellenmendonca01@gmail.com
}

\begin{abstract}
RESUMO - Um dos equipamentos mais usados para a secagem é o secador rotatório, tambor cilíndrico dotado de suspensores, que ao girar promove o cascateamento do material particulado através da corrente gasosa. Assim, a quantidade de sólidos contidos nos suspensores de um secador é extremamente importante para a análise do desempenho destas unidades. O trabalho tem por objetivo avaliar a quantidade de sólidos no suspensor para dois tipos de partículas num tambor rotatório com seis suspensores de três segmentos sem a presença do ar de secagem. As partículas usadas no trabalho foram esferas de vidro e fertilizante SSPG com 2,56 mm de diâmetro. Para o auxílio à compreensão do escoamento, utilizou-se a fluidodinâmica computacional (CFD), uma ferramenta cada vez mais utilizada dado o crescente aumento da capacidade de processamento dos computadores aliado ao alto custo de construção das plantas piloto. Utilizou-se o software FLUENT $^{\circledR}$ e a abordagem Euleriana Granular Multifásica para o estudo computacional. Foram realizadas comparações das frações de sólidos nos suspensores em função da posição angular tanto no trabalho experimental quanto de simulação e analisou-se o efeito do ângulo de repouso estático do sólido no escoamento.
\end{abstract}

\section{INTRODUÇÃO}

Os secadores rotatórios com suspensores são encontrados em diversos setores industriais, como indústrias de cimento, fertilizantes e minerais. Isso ocorre por causa da sua alta capacidade de processamento e flexibilidade de operação, o que faz com que esses sejam os mais utilizados dentre todos os tipos de secadores (Sheehan et al., 2005). O equipamento consiste de um tambor cilíndrico, inclinado por um pequeno ângulo com relação à horizontal, que gira em torno do seu próprio eixo. Internamente ao tambor se encontram suspensores acoplados, que promovem o cascateamento das partículas.

A descrição da fluidodinâmica dos secadores no estudo deste equipamento é fundamental, a qual pode ser realizada através da compreensão dos perfis de descarga de sólidos dos suspensores. Para isso, são utilizados tambores rotatórios com suspensores, mas sem a presença do ar de secagem, facilitando o entendimento do movimento das partículas. 


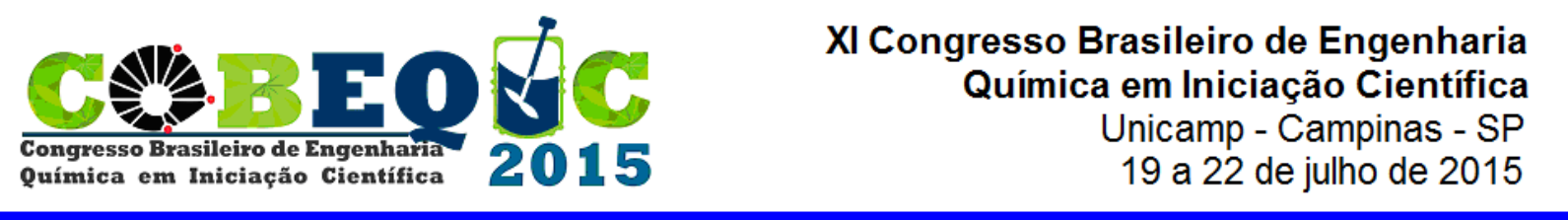

Alguns trabalhos experimentais já foram desenvolvidos para a compreensão do movimento das partículas nos secadores, porém, com o avanço da tecnologia e a crescente capacidade de processamento dos computadores, juntamente com os altos custos envolvidos na construção de plantas piloto, estão disponíveis ferramentas, como a fluidodinâmica computacional (CFD), que permite a abordagem de modelos matemáticos para o entendimento de fenômenos físicos. Trabalhos experimentais aliados com estudos em CFD estudando essa característica do escoamento são, então, muito importantes na caracterização do escoamento. Contudo, os estudos de CFD não eliminam o trabalho experimental, já que eles devem ser validados experimentalmente.

Então, este trabalho tem como objetivo geral comparar os valores de massa de sólidos dos suspensores das esferas de vidro e fertilizantes SSPG nos suspensores em diferentes posições angulares obtidos nos trabalhos experimentais, analisando o efeito do ângulo de repouso estático do sólido no escoamento, assim como comparar com os resultados estimados nas simulações utilizando a abordagem Euleriana Granular Multifásica.

\section{METODOLOGIA}

\subsection{Metodologia Experimental}

O equipamento utilizado no trabalho experimental consiste de um tambor cilíndrico com $0,108 \mathrm{~m}$ de diâmetro e $0,5 \mathrm{~m}$ de comprimento, fabricado em aço inox, colocado na horizontal, como mostra a Figura 1. Na parte interna do tambor existem seis suspensores igualmente espaçados com três segmentos cada. Cada segmento do suspensor possui o comprimento de 10, 4 e $4 \mathrm{~mm}$, sendo que o maior segmento está soldado à parede do tambor. Na extremidade frontal do tambor (Figura 1 (a)), foi colocado um visor de vidro temperado que permite fotografar a posição angular do suspensor e na outra extremidade do tambor foi montado um sistema em acrílico com o coletor de partículas.

Figura 1 - Foto da unidade experimental: (a) vista lateral e (b) vista frontal.

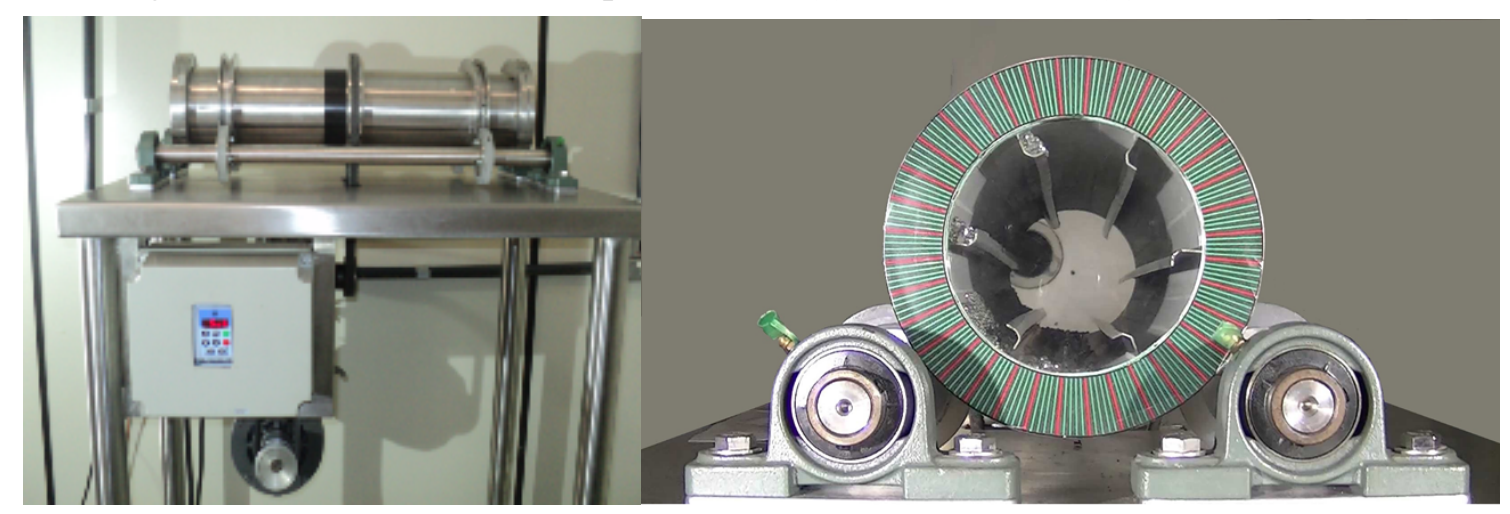

As partículas utilizadas no experimento foram esferas de vidro com 2,6 mm de diâmetro, massa específica de $2455 \mathrm{~kg} / \mathrm{m}^{3}$ e porosidade 0,37 e o fertilizante SSPG de mesmo diâmetro de peneira com massa específica bulk de $2090 \mathrm{~kg} / \mathrm{m}^{3}$ e porosidade do leito consolidado de 0,51. Utilizou-se cargas de partículas que correspondiam a $6 \%$ do volume do tambor, para que ele estivesse sobrecarregado, e a velocidade de rotação utilizada foi de $3 \mathrm{rpm}$. 


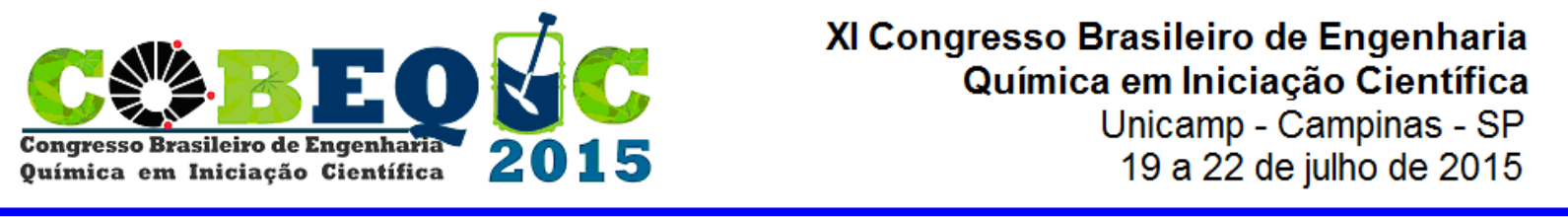

Procedimento experimental: Adiciona-se a massa de partículas previamente determinada e inicia-se o movimento de rotação. Quando o suspensor em interesse atinge determinada posição angular, interrompe-se a rotação do cilindro e em seguida fotografa-se a posição angular do suspensor. Para medi-la, o tambor possui um transferidor, o qual permite que as retas horizontal e vertical sejam traçadas. Utilizando o software Image $\mathrm{J} 囚$, mede-se a posição angular, que é o ângulo formado pela reta que vai da ponta do suspensor até a origem e a reta horizontal. Posteriormente, retira-se a tampa da extremidade em acrílico e introduz-se o coletor de partículas. Realiza-se então a coleta das partículas, com o tambor inclinado para que não haja perda de massa, e mede-se a massa nesta posição.

\subsection{Metodologia Numérica}

Utilizou-se o software FLUENT ${ }^{\circledR}$ 14.0, e o modelo Granular Euleriano Multifásico. A modelagem levou em consideração duas fases em que o ar foi representado como primária, e as esferas de vidro como secundária. Utilizou-se também, como parte da modelagem, a viscosidade friccional, através do modelo de Schaeffer (1987), que é função do ângulo de friç̧ão interno partícula-partícula, medido através de um plano inclinado, como descrito em Santos et al. (2013). Para as esferas de vidro deste trabalho, o ângulo de fricção interno medido foi de $28^{\circ}$ e para 0 fertilizante SSPG foi de $41^{\circ}$. Toda a modelagem detalhada do escoamento das partículas, assim como o equacionamento dos modelos utilizados, adotada neste trabalho pode ser encontrada no trabalho desenvolvido por Nascimento et al. (2014).

Estimativa de massa de sólidos: Devido à massa de sólidos nos suspensores ser importante para descrever o perfil de descarga do suspensor, foi desenvolvida uma metodologia para estimar a medida de sólidos nos suspensores através dos resultados, como a Figura 2, obtidos na simulação.

Figura 2 - Exemplo de perfil de escoamento gerado na simulação CFD.

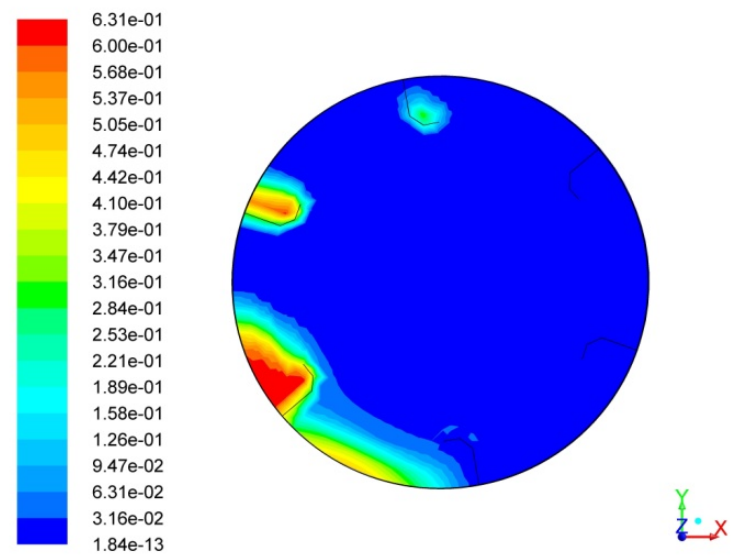

Os perfis gerados na simulação apresentam uma escala com 20 cores, em que cada cor representa uma faixa de concentrações volumétricas. Diferente da simulação, o resultado experimental não apresentou uma faixa de concentrações volumétricas em função da posição angular, dado que essa escala de cores é uma conseqüência de interpolações realizadas durante o cálculo numérico. Para calcular a massa de sólidos no suspensor, a massa de cada cor foi estimada através da multiplicação da área de cada cor pela média da fração volumétrica para a cor correspondente, pela massa específica da partícula e pelo comprimento 


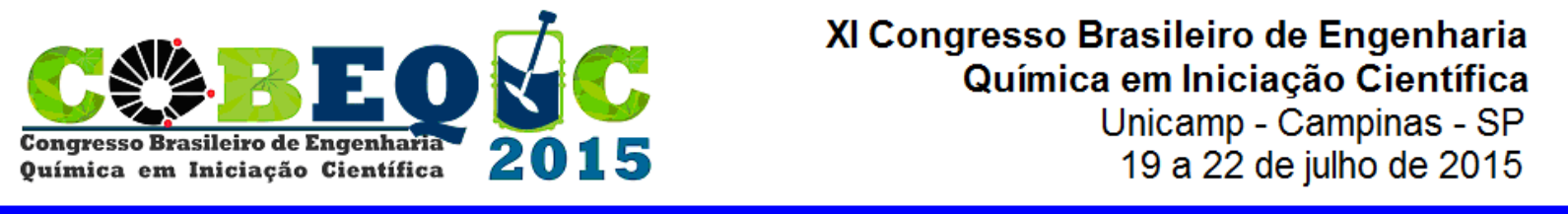

do tambor. O holdup (carga de sólidos) no suspensor é a soma das massas de cada cor, assim como mostra a Equação 1.

$$
h=\sum_{i=1}^{20} a_{i}\left(\frac{\alpha_{\max , \mathrm{i}}+\alpha_{\min , \mathrm{i}}}{2}\right) \rho_{S} L
$$

Em que $h$ é a massa de sólidos no suspensor, $a_{i}$ é a área correspondente a cada cor, $\alpha_{\text {max,i }}$ e $\alpha_{\text {min,i }}$ são respectivamente a máxima e a mínima fração de sólidos correspondente à cor, $\rho_{S}$ é a massa específica do sólido e $L$ é o comprimento do tambor.

\section{RESULTADOS E DISCUSSÃO}

Um dos objetivos deste trabalho é comparar o comportamento de dois tipos de partículas dentro de um tambor rotatório com suspensores. A primeira comparação feita entre os dois materiais foi a medida experimental da massa de sólidos em várias posições angulares. Analisando a Figura 3, observa-se uma maior quantidade de massa carregada pelo suspensor para a partícula cuja densidade é superior. Além disso, nota-se que as massas de esferas de vidro são bem maiores que as de fertilizante nas menores posições angulares, porém no final do descarregamento essa diferença se encontra irrelevante, isto se deve ao fato do material de vidro escoar menos, devido ao seu ângulo de repouso estático ser inferior ao do Fertilizante.

Figura 3 - Comparação entre os valores de massa de sólidos obtidos experimentalmente em função da posição angular.

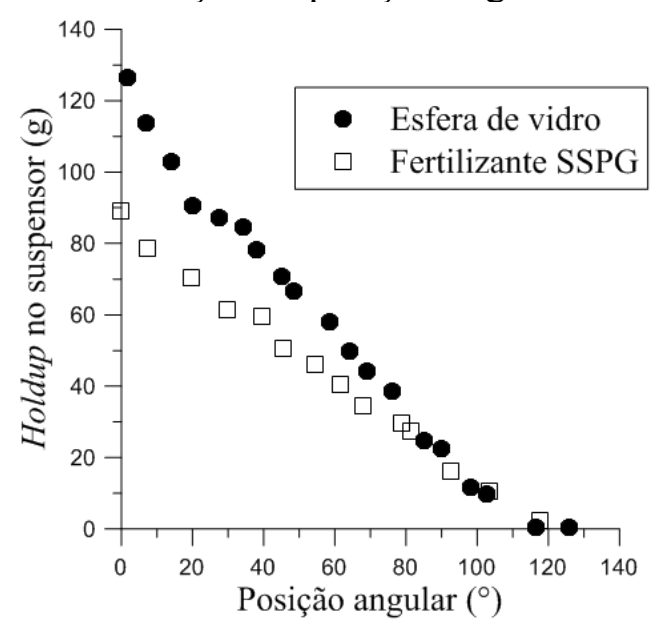

A aplicação do CFD para descrever o cascateamento do material dentro de um tambor rotatório requer um pouco mais de precisão pois, de acordo com Lee e Sheehan (2010), o descarregamento de sólidos do suspensor não é contínuo e isso deixa a fluidodinâmica envolvida no processo mais complexa. A parte que envolve a modelagem, assim como a investigação do modelo que mais se aproxima do experimental, podem ser encontradas em Nascimento et al. (2014). 
Na Figura 4 observa-se que os resultados de simulação estiveram bem próximos dos experimentais para os dois materiais estudados, mostrando que o modelo utilizado consegue prever bem o escoamento dos sólidos. Comparando as Figuras 4(a) e 4(b), verificou-se que a simulação também conseguiu prever aproximadamente a mesma diferença de massa nos primeiros ângulos entre as duas partículas.

Figura 4- Comparação entre os valores de massa de sólidos obtidos experimentalmente e via CFD para: (a) esferas de vidro e (b) Fertlizante SSPG.

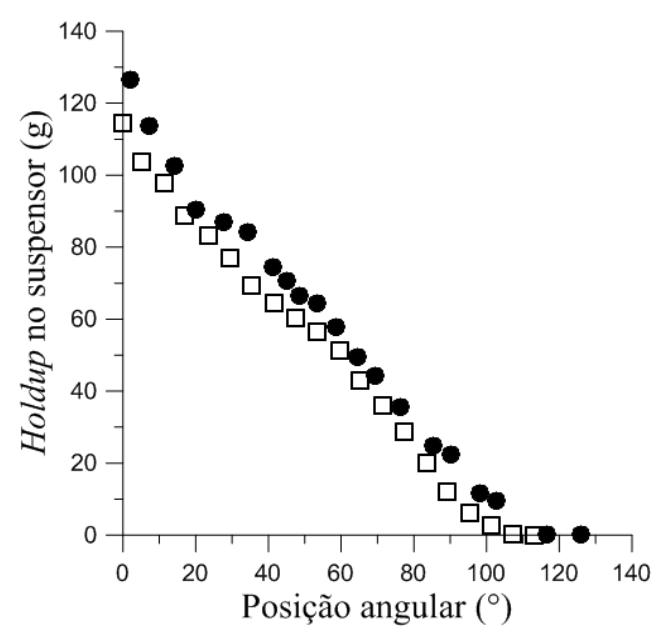

(a)

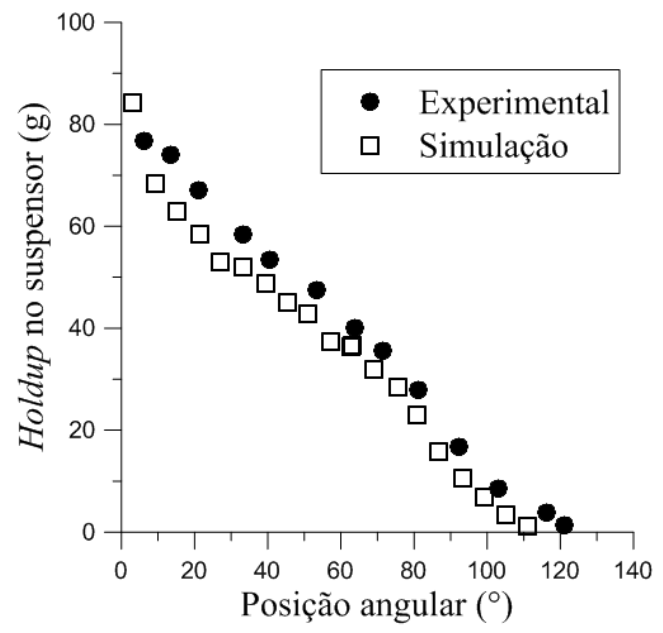

(b)

Como o volume colocado no experimento foi o mesmo para as duas partículas, observou-se que a análise da porcentagem volumétrica em função da posição angular para os dois materiais poderia explicar melhor a influência do ângulo de repouso estático no tambor, sendo que seriam eliminados a influencia das diferenças dos valores de densidade, já observados na Figura 3. Os resultados estão apresentados nas Figuras 5(a) e 5(b).

Figura 5- Comparação entre os valores de porcentagem volumétrica obtidos:

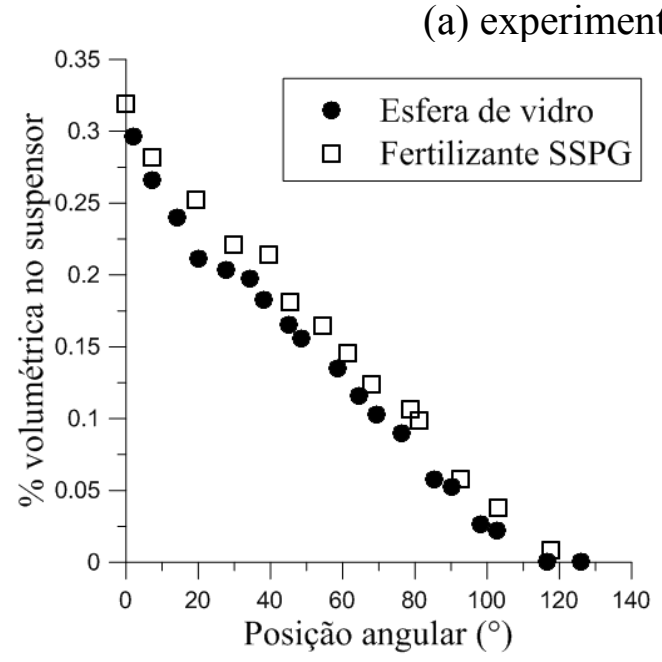

(a)

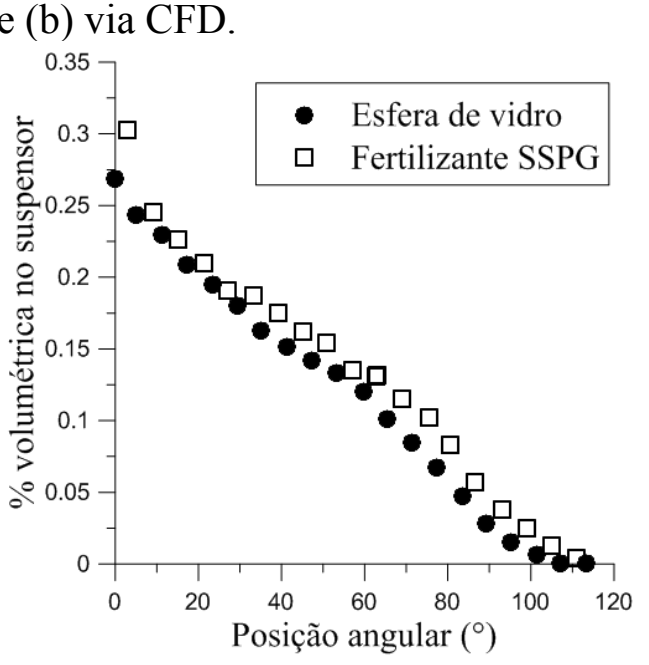

(b) 
Observa-se na Figura 5 (a), que os suspensores carregam praticamente o mesmo volume de partículas e essas pequenas alterações decorreram do coeficiente de atrito dinâmico que depende do ângulo de repouso estático. Além disso, observou-se na Figura 5 (b) que os resultados da simulação se apresentaram novamente bem próximos dos resultados encontrados experimentalmente, com desvios de no máximo 12 gramas. Apesar disso, novos modelos de viscosidade granular devem ser estudados visando diminuir tais desvios.

\section{CONCLUSÃO}

Os resultados experimentais permitiram analisar a influência do ângulo de repouso estático na quantidade de sólidos nos suspensores, sendo que quanto maior esse ângulo, mais coeso o material, e consequentemente a sua taxa de descarga do suspensor é menor. Analisando a porcentagem volumétrica em função da posição angular, observou-se que estando o tambor carregado com o mesmo volume, os suspensores tendem a carregar praticamente a mesma quantidade volumétrica de sólido, sendo que algumas pequenas alterações decorrem do coeficiente de atrito dinâmico que depende do ângulo de repouso estático.

Observando os resultados encontrados no trabalho experimental e na simulação CFD, concluiu-se que o modelo encontrado foi adequado tanto para a predição da fração de sólidos quanto de porcentagem volumétrica no suspensor, apesar da necessidade de investigação de diferentes modelos de viscosidade granular. Porém, a simulação via CFD com o modelo utilizado se mostrou uma ferramenta promissora para o estudo de perfis de descarga de sólidos em tambor rotatório com suspensores.

\section{REFERÊNCIAS}

LEE, A.; SHEEHAN, M.E. Development of a geometric flight unloading model for flighted rotary dryers. Powder Technology, v.198, p.395-403, 2010.

NASCIMENTO, S.M.; CAMPOS, G. A. S.; LIMA, D. M.; ÁVILA, L. F. G.; BARROZO, M. A .S.; DUARTE, C. R. Estudo experimental e de simulação CFD da dinâmica de partículas em tambores rotatórios com suspensores. XX Congresso Brasileiro de Engenharia Química. Anais do congresso, 2014.

SANTOS, D.A.; PETRI, I.J.; DUARTE, C.R.; BARROZO, M.A.S. Experimetal and CFD study of the hydrodynamic behavior in a rotating drum. Powder Technology, v. 250, p. 52-62, 2013.

SCHAEFFER, G., Instability in the evolution equations describing incompressible granular flow, J. Differential Equations, vol. 66, p. 19-50, 1987.

SHEEHAN, M.E.; BRITTON, P.F.; SCHNEIDER, P.A. A model for solids transport in flighted rotary dryers based on physical considerations. Chem. Eng. Sci. v.60, p. 41714182, 2005. 